\title{
THE INFLUENCE OF NONUNIFORM CLOUD COVER ON TRANSIT TRANSMISSION SPECTRA
}

\author{
Michael R. Line ${ }^{1,2,3,4,5}$ and Vivien Parmentier ${ }^{4,6}$ \\ ${ }^{1}$ NASA Ames Research Center, Moffet Field, CA 94035, USA \\ ${ }^{2}$ Bay Area Environmental Research Institute, 625 2nd Street Ste. 209, Petaluma, CA 94952, USA \\ ${ }^{3}$ School of Earth and Space Exploration, Arizona State University, 781 S Terrace Road, Tempe, AZ 85281, USA; mrline@ucsc.edu \\ ${ }^{4}$ Department of Astronomy \& Astrophysics, University of California-Santa Cruz, 1156 High Street, Santa Cruz, CA 95064, USA \\ Received 2015 November 30; accepted 2016 February 8; published 2016 March 21
}

\begin{abstract}
We model the impact of nonuniform cloud cover on transit transmission spectra. Patchy clouds exist in nearly every solar system atmosphere, brown dwarfs, and transiting exoplanets. Our major findings suggest that fractional cloud coverage can exactly mimic high mean molecular weight atmospheres and vice versa over certain wavelength regions, in particular, over the Hubble Space Telescope (HST) Wide Field Camera 3 (WFC3) bandpass $(1.1-1.7 \mu \mathrm{m})$. We also find that patchy cloud coverage exhibits a signature that is different from uniform global clouds. Furthermore, we explain analytically why the "patchy cloud-high mean molecular weight" degeneracy exists. We also explore the degeneracy of nonuniform cloud coverage in atmospheric retrievals on both synthetic and real planets. We find from retrievals on a synthetic solar composition hot Jupiter with patchy clouds and a cloud-free high mean molecular weight warm Neptune that both cloud-free high mean molecular weight atmospheres and partially cloudy atmospheres can explain the data equally well. Another key finding is that the HST WFC3 transit transmission spectra of two well-observed objects, the hot Jupiter HD 189733b and the warm Neptune HAT-P-11b, can be explained well by solar composition atmospheres with patchy clouds without the need to invoke high mean molecular weight or global clouds. The degeneracy between high molecular weight and solar composition partially cloudy atmospheres can be broken by observing the molecular Rayleigh scattering differences between the two. Furthermore, the signature of partially cloudy limbs also appears as a $\sim 100 \mathrm{ppm}$ residual in the ingress and egress of the transit light curves, provided that the transit timing is known to seconds.
\end{abstract}

Key words: methods: analytical - planets and satellites: atmospheres - planets and satellites: composition planets and satellites: individual (HD 189733b, HAT-P-11b)

\section{INTRODUCTION}

Clouds are ubiquitous in our solar system, brown dwarfs, and extrasolar planets. They often complicate the interpretation of spectra owing to our ignorance of their properties. This can strongly inhibit our ability to place precision constraints on other atmospheric properties like temperatures and abundances (Ackerman \& Marley 2001; Kirkpatrick 2005; Burrows et al. 2006; Cushing et al. 2008; Howe \& Burrows 2012; Morley et al. 2013; 2015; Knutson et al. 2014; Kreidberg et al. 2014a; 2015; Marley \& Robinson 2014; Vahidinia et al. 2014; Benneke 2015). In particular, they strongly impact transit transmission spectra, because the longer optical path lengths permit a greater sensitivity to trace cloud species (Fortney 2005). To date, only one-dimensional, limb-averaged cloud models have been used to interpret the transmission spectra of exoplanets (Fortney et al. 2010; Howe \& Burrows 2012; Morley et al. 2013; 2015; Fraine et al. 2014; Kreidberg et al. 2014a, 2015; Benneke 2015). In this investigation we show that the presence of inhomogeneous clouds along the terminators of transiting exoplanets can strongly influence our interpretation of current transit transmission spectra.

Inhomogeneous clouds are common in our solar system over a broad range of bulk planetary properties (terrestrial, Jovian, cold, hot, etc.). Meridional atmospheric cells combined with vertical temperature gradients create the banded clouds seen on Earth and on Jupiter; warm, humid parcels of gas rise from one

\footnotetext{
5 NASA Hubble Postdoctoral Fellow.

${ }^{6}$ NASA Sagan Postdoctoral Fellow.
}

latitude in the atmosphere where they then cross the dew point temperature, at which point condensates form. The now dry air then sinks back down, leading to clear skies at a different latitude. Similar processes are presumed to happen in brown dwarf atmospheres (Burgasser et al. 2002; Marley et al. 2010; Zhang \& Showman 2014), where observations are strongly suggestive of nonuniform cloud cover (Buenzli et al. 2012, 2014; Radigan et al. 2012, 2014; Apai et al. 2013; Crossfield et al. 2014; Metchev et al. 2015).

In tidally locked exoplanets, two mechanisms have been identified to form inhomogeneous clouds. First, the meridional circulation can transport condensate material from the equatorial regions to the polar regions, a phenomenon suggested to be common in hot Jupiters and sub-Neptune planets (Parmentier et al. 2013; Charnay et al. 2015a). The second mechanism is specific to the hottest of the tidally locked planets. The intense and inhomogeneous stellar irradiation they receive creates a large day-night temperature contrast that drives a strong westto-east atmospheric circulation pattern. This atmospheric circulation, dominated by a superrotating equatorial jet, advects thermal energy eastward, leading to a strong west-to-east terminator temperature gradient of several hundreds of degrees. This eastward shift in temperature was first predicted by Showman \& Guillot (2002) and has since been observed in a number of hot Jupiters (Knutson et al. 2007, 2009, 2012; Crossfield et al. 2010; Cowan et al. 2012; Lewis et al. 2013; Stevenson et al. 2014). Such a large horizontal temperature gradient can lead to longitudinally varying cloud cover as numerous condensable species can be in a condensed state on the west limb but gaseous in the east limb. Recent phase curve observations in visible light from the Kepler spacecraft are 
strongly suggestive of inhomogeneous dayside cloud coverage, with cloudy western daysides and clear eastern daysides (Demory et al. 2013; Esteves et al. 2015; Hu et al. 2015; Parmentier et al. 2015, submitted; Shporer \& Hu 2015), very much in agreement with expectations.

In the remainder of the paper we describe how a nonuniform cloud cover along the planetary terminator can influence the observed transit transmission spectra and how failing to account for nonuniform cloud cover can bias molecular abundance determinations. In Section 2 we illustrate the basic idea and describe the impact that nonuniform terminator cloud cover can have on transit transmission spectra. Section 3 reviews, analytically, the basic mechanisms that control the shape of transit transmission spectra and the role of nonuniform terminator cloud cover. In Section 4 we show quantitatively, via atmospheric retrievals, how nonuniform cloud cover can impact water abundance determinations on both synthetic data and two well-observed planets, the hot Jupiter HD 189733b and warm Neptune HAT-P-11b. In Section 5 we show how nonuniform cloud cover can present itself as residuals in transit light curves. Finally, we summarize our findings and discuss caveats and implications in Section 6.

\section{BASIC CONCEPT AND IMPACT ON TRANSIT TRANSMISSION SPECTRA}

We use a transit transmission forward model (Line et al. 2013; Kreidberg et al. 2014b, 2015; Swain et al. 2014) to generate a variety of spectra over the Hubble Space Telescope (HST) Wide Field Camera 3 (WFC3) bandpass to illustrate the basic concept. The model numerically solves the equations described in Brown (2001) and Tinetti et al. (2012). The inputs are the scale height isothermal temperature $(T)$, the planetary radius at 10 bars, the opaque (in the limb geometry) gray cloud top pressure $\left(P_{c}\right)$, a terminator cloud fraction $(f)$, and the gas abundances. For simplicity we include only water as the gaseous absorber as it has been the only molecule robustly detected over the WFC3 bandpass (e.g., Deming et al. 2013; Kreidberg et al. 2014a, 2015). The remaining "filler" gas is assumed to be a mixture of molecular hydrogen and helium in solar proportions. Clouds are modeled rather simplistically assuming that no light is transmitted through the atmosphere at pressures deeper than $P_{c}$. Nonuniform cloudy transmission spectra are computed via a linear combination of a globally clear atmosphere and globally cloudy atmosphere (similar to Marley et al. 2010; Morley et al. 2014 for brown dwarf emission spectra) using the following:

$$
\alpha_{\lambda, f}=f \alpha_{\lambda, \text { cloudy }}+(1-f) \alpha_{\lambda, \text { clear }},
$$

where $\alpha_{\lambda, f}$ is the wavelength-dependent eclipse depth for cloud fraction $f$ and $\alpha_{\lambda \text {,cloudy }}$ and $\alpha_{\lambda \text {,clear }}$ are the wavelength-dependent eclipse depths for a globally cloudy and globally clear atmosphere, respectively. This is effectively a "two-dimensional" model as we do not consider variations along the tangent rays (unlike in Burrows et al. 2010; Fortney 2010). Figure 1 illustrates the basic concept for two representative planets, a hot Jupiter and a warm Neptune. The necessary planet and atmosphere parameters are shown in Table 1.

For this setup we use a relatively high altitude opaque cloud in order to substantially flatten the spectra, a reasonable cloud top pressure in lieu of recently observed flat transmission spectra (Morley et al. 2013, 2015; Knutson et al. 2014;
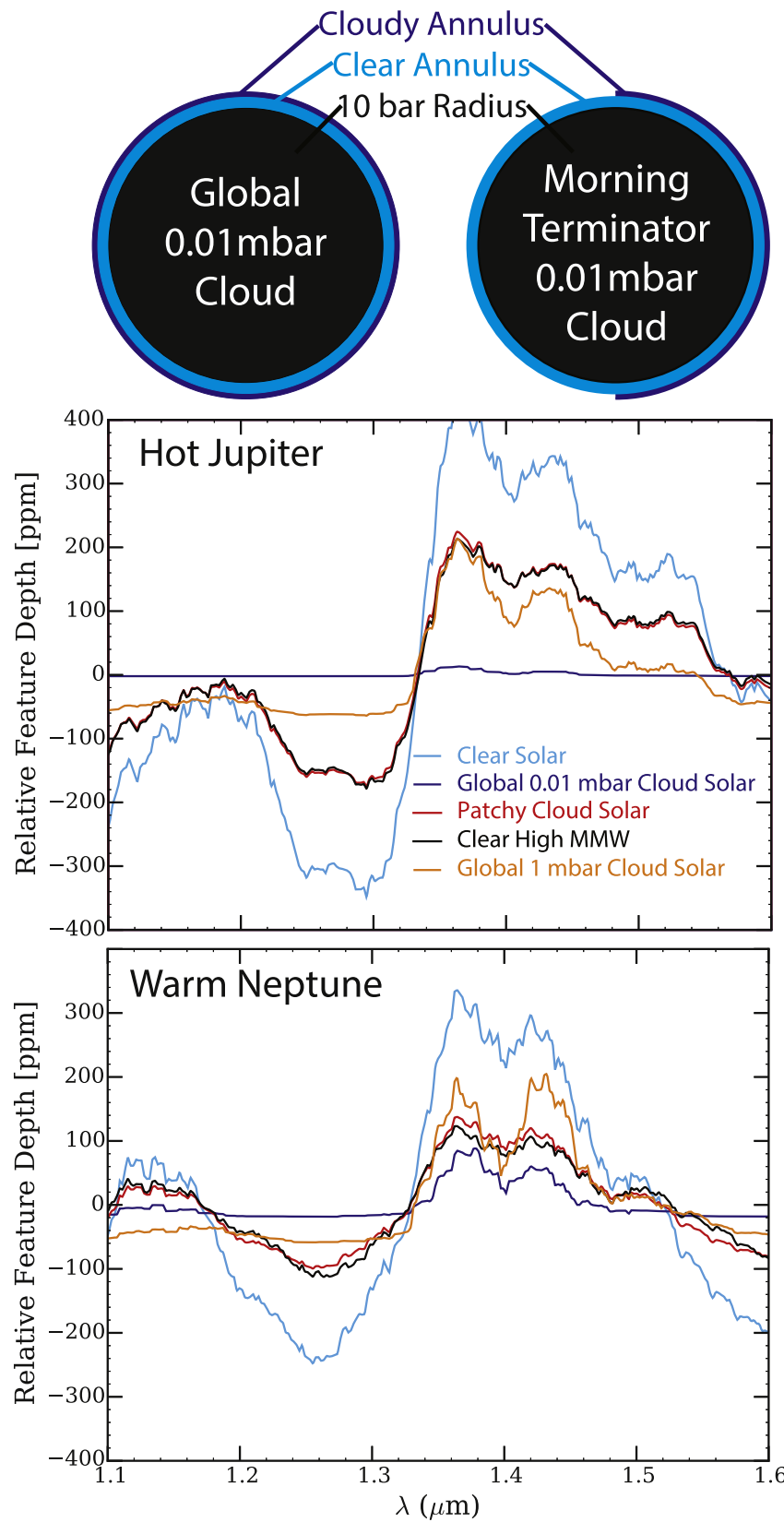

Figure 1. Impact of nonuniform cloud cover on a hot-Jupiter and warmNeptune transit transmission spectrum. The cartoon at the top illustrates the relative change in radius (to scale for the hot Jupiter) due to the the clear and cloudy annuli. In the bottom two panels we compare representative spectra of different commonly encountered atmospheric scenarios for a hot Jupiter (middle) and warm Neptune (bottom) (see Table 1) with partial cloudy spectra. The spectra are offset to have zero mean. Note the near identical match between the patchy cloud and high mean molecular weight (mmw) spectra.

Kreidberg et al. 2014a) and General Circulation Model (GCM) tracer studies (Parmentier et al. 2013; Charnay et al. 2015b). We see that the nonuniform cloud cover damps the spectral features as it is an average of a near flat line and a clear atmosphere. Certainly different cloud distributions (e.g., a cloudy northern hemisphere, clear southern hemisphere) could have the same terminator cloud fraction. For a given cloud fraction, we would not be able to determine the spatial distribution of clouds along the terminator; however, this 
Table 1

Parameters Used to Generate the Simulated Representative Planet Atmospheres Shown in Figure 1

\begin{tabular}{|c|c|c|}
\hline Parameter & Hot Jupiter & Warm Neptune \\
\hline Planet radius at 10 bars $\left(R_{\text {Jupiter }}\right)$ & 1.25 & 0.38 \\
\hline Stellar radius $\left(R_{\text {Sun }}\right)$ & 1.15 & 0.46 \\
\hline Planet gravity (m/s) & 10 & 12.6 \\
\hline Isothermal temperature $(\mathrm{K})$ & 1500 & 700 \\
\hline Solar water mixing ratio ${ }^{a}$ & $4.5 \times 10^{-4}$ & $6.3 \times 10^{-4}$ \\
\hline High-metallicity water mixing ratio ${ }^{b}$ & $1.5 \times 10^{-1}(250 \times$ solar $)$ & $3.0 \times 10^{-1}(500 \times$ solar $)$ \\
\hline High-metallicity mean molecular weight $(\mathrm{amu})^{\mathrm{c}}$ & 4.6 & 7 \\
\hline Global cloud top pressure $(\mathrm{mbar})^{\mathrm{d}}$ & 1 & 1 \\
\hline Patch cloud top pressure (mbar) & 0.01 & 0.01 \\
\hline Patchy cloud fraction ${ }^{\mathrm{e}}$ & 0.5 & 0.6 \\
\hline
\end{tabular}

Notes.

a Thermochemical equilibrium abundance of water at the isothermal temperature and 1 mbar using solar elemental abundances.

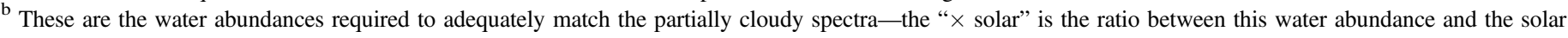
water abundance, and not the elemental abundances.

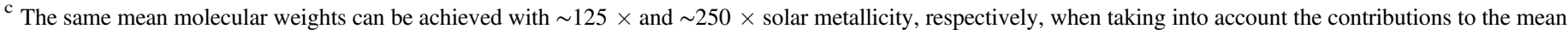
molecular weight from other molecules.

${ }^{\mathrm{d}}$ For the clear atmospheres, this is set to the deepest pressure layer in the model grid, 30 bars.

${ }^{\mathrm{e}}$ Fraction of terminator covered in the 0.01 mbar clouds. This is set to 1 for the global clouds and 0 for clear atmospheres.

degeneracy may be broken through high-precision transit light curves (Section 5).

The shape of the nonuniform cloud cover spectra is much different than a globally uniform deeper cloud (red curve versus green curve). A globally uniform deeper cloud has a flatter shape $\leqslant 1.32 \mu \mathrm{m}$ and a somewhat deeper trough near the peak at $1.4 \mu \mathrm{m}$ and a steeper slope $>1.36 \mu \mathrm{m}$. The most interesting result from this exercise is the near-perfect match between the nonuniform cloud cover (red) and the high mean molecular weight ${ }^{7}$ (typically assumed to be due to high metallicity) clear spectrum (black) for both planet setups. The degeneracy between uniform clouds and high mean molecular weight has already been investigated in a variety of planets (Benneke \& Seager 2013; Fraine et al. 2014; Knutson et al. 2014). The nonuniform cloud degeneracy is different as the shape and the amplitude of the higher mean molecular weight spectral features in the WFC3 bandpass can be almost exactly reproduced by the nonuniform cloud cover, but less so by a globally uniform cloud. In the next section we describe, analytically, what controls the shape of spectral features in transmission and why nonuniform cloud cover exactly mimics high mean molecular weight atmospheres.

\section{WHAT CONTROLS THE SHAPE OF TRANSMISSION SPECTRA?}

Here we discuss the variables that control the shape of spectral features in transit transmission spectra within the simple analytic formulation presented by Lecavelier Des Etangs et al. (2008).

\subsection{One Absorber}

The original Lecavelier Des Etangs et al. (2008) formulation (see their Equation (1)) computes the equivalent wavelengthdependent sharp occulting disk radius, $R_{p}+z_{\lambda}$ (where $R_{p}$ is

\footnotetext{
7 By high mean molecular weight we mean high enough to begin to shrink spectral features, which in the scenarios in this paper begins around a molecular weight of 2.8 (see also Benneke \& Seager 2013). Such a molecular weight for typical solar elemental proportions in hydrogen-dominated atmospheres occurs near metallicities of $\sim 60 \times$ solar.
}

wavelength independent) for a single absorber with

$$
z_{\lambda}=\frac{k_{b} T}{\mu g} \ln \left(\xi \sigma_{\lambda} \frac{1}{\sqrt{k_{b} T \mu g}} \beta\right)
$$

and

$$
\beta=\frac{P_{0}}{\tau_{e q}} \sqrt{2 \pi R_{p}},
$$

where $k_{b}$ is Boltzman's constant, $T$ is temperature, $\mu$ is the atmospheric mean molecular weight, $g$ is gravity, $\xi$ is the volume mixing ratio of a particular absorber with cross section $\sigma_{\lambda}, P_{0}$ is the reference pressure at " 0 altitude," and $\tau_{\text {eq }}$ is the optical depth required to produce and equivalent opaque disk. The wavelength-dependent eclipse depth $\left(\alpha_{\lambda}\right)$ can then be given with $\left(\left(R_{p}+z_{\lambda}\right) / R_{\text {star }}\right)^{2}$. If we assume that the atmospheric thickness is much smaller than the planet radius, we can approximate the eclipse depth as

$$
\alpha_{\lambda} \approx\left(\frac{R_{p}}{R_{\mathrm{star}}}\right)^{2}+\frac{2 R_{p} z_{\lambda}}{R_{\mathrm{star}}^{2}} .
$$

We define the spectral shape as the wavelength-dependent slope, $d \alpha_{\lambda} / d \lambda$. Differentiating Equation (4) with respect to $\lambda$ gives

$$
\frac{d \alpha_{\lambda}}{d \lambda}=\frac{2 R_{p}}{R_{\mathrm{star}}^{2}} H \frac{d \ln \left(\sigma_{\lambda}\right)}{d \lambda},
$$

where $\frac{k_{b} T}{\mu g}$ is the scale height, $H$, similar to Equation (2) in Lecavelier Des Etangs et al. (2008). From this we see that for a given planet $\left(R_{p}\right.$ and $R_{\text {star }}$ ) the spectral shape depends on the wavelength dependence of the absorption cross section and the scale height.

Note how the volume mixing ratio of the absorber, $\xi$, does not appear in this relation. This suggests that given a single spectrally dominant trace species over some wavelength range, the spectral shape is independent of the molecular abundance. As a consequence, it is not possible to measure the molecular abundance (Benneke \& Seager 2012; Griffith 2014), unless 
using the small pressure dependence of absorption cross section (de Wit \& Seager 2013). This breaks down when the abundance of the trace species is large enough to impact the mean molecular weight or when an additional absorber is present.

\subsection{Multiple Absorbers}

We now ask how the spectral shape depends on contributions from multiple absorbers. This will be important for understanding the contribution of clouds to the spectral shape. We begin by generalizing the above equations for $N$ absorbers, $i$, with

$$
z_{\lambda}=H \ln \left(\frac{1}{\sqrt{k_{b} T \mu g}} \beta \sum_{i}^{N} \xi_{i} \sigma_{\lambda, i}\right)
$$

and the spectral shape,

$$
\frac{d \alpha_{\lambda}}{d \lambda}=\frac{2 R_{p}}{R_{\text {star }}^{2}} H \frac{1}{\sum_{i}^{N} \xi_{i} \sigma_{\lambda, i}} \sum_{i}^{N} \xi_{i} \frac{d \sigma_{\lambda, i}}{d \lambda} .
$$

For the remainder of the analysis lets consider only two opacity sources with absorption cross section $\sigma_{1}$ and abundance $\xi_{1}$ for absorber 1 and $\sigma_{2}$ and abundance $\xi_{2}$ for absorber 2 . Equation (7), after some manipulation, then becomes

$$
\frac{d \alpha_{\lambda}}{d \lambda}=\frac{2 R_{p}}{R_{\text {star }}^{2}} H \frac{1}{1+\frac{\xi_{2} \sigma_{\lambda, 2}}{\xi_{1} \sigma_{\lambda, 1}}}\left(\frac{d \ln \sigma_{\lambda, 1}}{d \lambda}+\frac{\xi_{2} \sigma_{\lambda, 2}}{\xi_{1} \sigma_{\lambda, 1}} \frac{d \ln \sigma_{\lambda, 2}}{d \lambda}\right) .
$$

This shows that, in addition to the scale height modulating the spectral slope, we now have to consider the shape modulation due to the relative contributions of the different absorbers. Provided that there are no other dependencies, only the ratios of abundances can be measured (Benneke \& Seager 2012). In a clear, solar composition hot Jupiter atmosphere the dominant absorber contributions will come from water, molecular Rayleigh scattering, and the $\mathrm{H}_{2}-\mathrm{H}_{2} / \mathrm{He}$ collision induced absorption (CIA). If a feature is attributed to CIA and another to water, then the water-to- $\mathrm{H}_{2} / \mathrm{He}$ abundance ratio can be measured. That ratio, combined with the idea that all mixing ratios have to sum to unity, permits the absolute abundance determinations (Benneke \& Seager 2012). This is effectively what sets a lower bound on the water abundance. Identifying spectral regions where the cross-section contrast is minimized (e.g., $\sigma_{\lambda, 1} \approx \sigma_{\lambda, 2}$ ) would permit a stronger sensitivity to the trace absorber as the slopes in Equation (8) are more balanced. If a feature is attributed to clouds and another to water, then there is a strong uncertainty on the water abundance because of our lack of priors on the cloud properties. CIA and gray clouds are distinguishable in the WFC3 bandpass because the CIA has a nongray opacity structure (see Figure 6 in Line et al. 2013).

Now we assume that the two absorbers are water and a gray cloud uniformly distributed through the atmosphere. ${ }^{8}$ The gray cloud cross section, by definition, has zero spectral slope, so

\footnotetext{
8 This analytic formulation assumes vertical homogeneity in the absorbers and thus does not permit the "hard cloud top pressure" parameterization used in many spectral interpretations, including all of the numerical simulations in this investigation. However, the results in this analytic discussion are appropriate as one can find an "equivalent" hard cloud top pressure that mimics the gray uniform absorber. Increasing the uniform cloud opacity has the same effect as decreasing the hard cloud top pressure.
}

Equation (8) reduces to

$$
\frac{d \alpha_{\lambda}}{d \lambda}=\frac{1}{1+\frac{\xi_{\mathrm{cld}} \sigma_{\lambda, \mathrm{cld}}}{\xi_{\mathrm{H} 2 \mathrm{O}} \sigma_{\lambda, \mathrm{H} 2 \mathrm{O}}}} \frac{2 R_{p}}{R_{\mathrm{star}}^{2}} H \frac{d \ln \left(\sigma_{\lambda, \mathrm{H} 2 \mathrm{O}}\right)}{d \lambda} .
$$

When the cloud opacity is low $\left(\xi_{\mathrm{H} 2 \mathrm{O}} \sigma_{\mathrm{H} 2 \mathrm{O}} \gg \xi_{\text {cld }} \sigma_{\mathrm{cld}}\right)$, Equation (9) reduces to Equation (5). If the opposite is true, then the spectral modulation approaches zero. Note how the prefactor multiplying the wavelength-dependent cross section is also wavelength dependent, unlike Equation (5). This means that an atmosphere with a gray global cloud with an opacity comparable to that of water (e.g., such that the spectral features are muted but not completely flat) cannot be exactly mimicked by a clear, smaller scale height atmosphere.

\subsection{Patchy Clouds}

Now we consider how the spectral shape is modulated by nonuniform terminator cloud cover assuming that the cloudy portion of the terminator is dominated by the cloud opacity. Combining Equation (1) with Equation (5) (the clear atmosphere spectral shape) and the assumption that the cloud opacity dominates over the molecular absorber opacity on the cloudy terminator (such that the spectral slope is zero), we obtain

$$
\frac{d \alpha_{\lambda}}{d \lambda}=(1-f) \frac{2 R_{p}}{R_{\mathrm{star}}^{2}} H \frac{d \ln \left(\sigma_{\lambda, \mathrm{H} 2 \mathrm{O}}\right)}{d \lambda} .
$$

This is effectively the same as Equation (5) for a clear atmosphere except that the prefactor that scales the wavelengthdependent cross section now depends on the terminator cloud fraction, $f$. From this, it can be readily seen that there is a degeneracy between scale height and terminator cloud fraction, as well as why a high mean molecular weight atmosphere can identically mimic a low mean molecular weight atmosphere with partial terminator cloud coverage. Note how with the partial cloudy atmosphere there is no wavelength dependence on the prefactor, in contrast to the global cloud (Equation (9)). This suggests, in theory, that partially cloudy and fully cloudy atmospheres are therefore distinguishable.

One may ask why we are sensitive to the mean molecular weight as opposed to the temperature in this cloud-scale height degeneracy. Technically, the scale height depends in exactly the same way on both the molecular weight and temperature (we assume gravity is well determined). Doubling the mean molecular weight has the same effect as halving the temperature on the scale height and hence spectral slope. However, the temperature affects not only the scale height but also the strength of the absorption cross sections. It is the temperature dependence of the absorption cross sections that permits us to disentangle temperature from molecular weight. Furthermore, our a priori knowledge of the planetary temperature from energy balance arguments is greater than that of the mean molecular weight (Benneke \& Seager 2012). That is, it is more physically plausible to increase the mean molecular weight of the atmosphere by an order of magnitude than the limb temperature. In the next section we quantitatively explore the patchy cloud degeneracy within an atmospheric retrieval framework. 


\section{IMPACT ON ATMOSPHERIC RETRIEVALS}

\subsection{Simulated Data}

We generate two representative synthetic WFC3 observations and explore the degeneracies introduced by partial cloud cover. The first is a $1500 \mathrm{~K}$ solar composition $(450 \mathrm{ppm}$ of water) hot Jupiter with a $50 \%$ terminator cloud fraction at 0.01 mbar (same as the scenario described in Section 2 and Table 1). We randomly draw a simulated set of observations with 50 ppm error bars and $0.02 \mu \mathrm{m}$ resolution (e.g., Kreidberg et al. 2014b). The second scenario is representative of the warm-Neptune class of planets (e.g., GJ 436b, HAT-P-11b). It is a $700 \mathrm{~K}$ high-metallicity $(500 \times$ the solar water abundance in thermochemical equilibrium-mean molecular weight of $\sim 7 \mathrm{amu}$ ) cloud-free atmosphere (cloud fraction 0, cloud depth at 30 bars) with $35 \mathrm{ppm}$ error bars (e.g., Kreidberg et al. 2014a). Figure 2 (top row) shows the simulated spectra for each scenario (black diamonds with error bars). Again, water is the only trace gas absorber. Similar feature sizes in the high mean molecular weight scenarios can be obtained with lower metallicities when taking into account the contribution to the mean molecular weight due to other heavier molecules.

We employ PyMultiNest ${ }^{9}$ (Buchner et al. 2014), a python wrapper to the commonly used MultiNest (Feroz et al. 2009) nested sampling algorithm, to explore the parameter degeneracies. Nested sampling algorithms are well suited for exploring multimodal and highly correlated parameter spaces (see, e.g., Benneke \& Seager 2013 for application to superEarth transmission spectra), as well as the efficient computation of the Bayesian evidence. Figure 2 summarizes the retrieved results. The bottom row shows the retrieved probability distributions for the interesting parameters (the scale height temperature and 10 bar radius are not shown for clarity). The water abundance histograms for both scenarios clearly have two modes. The first mode is the near-solar water abundance mode (solar mean molecular weight of 2.3) requiring nonuniform cloud cover. The second is a high water abundance (corresponding to a mean molecular weight of $>2.8$ ) mode requiring no cloud at all (e.g., only a lower-pressure, highaltitude limit).

To further explore this bi-modality, we isolate these two modes by drawing samples from the full posterior with water abundances that fall in either the high (blue) or low (red) water models (the cut being made at a $\log \left(\mathrm{H}_{2} \mathrm{O}\right)$ value of -1.5$)$. The median spectra from each of these modes are shown in the top row of Figure 2. In both observational scenarios we find that the low water mode requires fractional cloud coverage with approximately half of the terminator covered in high-altitude $(P<0.1$ mbar) clouds. In the high water abundance (high mean molecular weight) mode there is no longer a strong sensitivity to the location of the cloud (and correspondingly the cloud fraction), as the large limb optical depth obscures the presence of any cloud down to $\sim 0.1$ mbar pressures.

In order to be more quantitative, we compute the log of the Bayes factor $(\ln B)$ for the modes, a metric for quantitatively comparing two competing models (e.g., Cornish \& Littenberg 2007). For the hot-Jupiter scenario we obtain an $\ln B$ of 0.7 (considered negligible on the Jeffery's scale; Trotta 2008), suggesting that we cannot confidently distinguish the two modes, though the preference is slightly weighted toward the

\footnotetext{
http://johannesbuchner.github.io/PyMultiNest/index.html
}

high water/mean molecular weight abundance mode. For the high mean molecular weight warm-Neptune scenario the preference for one mode over the other is also negligible $(\ln B=0.5)$. These conclusions are unsurprising given that the median spectra (top row, Figure 2) for each mode are nearly identical (as expected given Figure 1).

For completeness we also ask the question of how well these spectra can be explained with a simple uniform cloud cover model with solar-like composition of water. To do this, we perform a separate retrieval where we place an upper bound on the water abundance prior at $\log \mathrm{H}_{2} \mathrm{O}=-1.5$. This effectively eliminates the possibility of the retrieval searching out the high mean molecular weight mode. Figure 2 (top row) shows the median spectra (in orange) from this setup. These spectra are substantially different from the patchy cloud or high mean molecular weight median spectra, with flatter features $<1.3 \mu \mathrm{m}$. The Bayesian evidence suggests a strong preference of the patchy cloud or high-metallicity scenarios over the globally cloudy solar-like composition $(\ln B$ of $\sim 7$ for the hot Jupiter and $\sim 5$ for the warm Neptune). This suggests that our synthetic observational setups can distinguish nonuniform cloud cover (or high molecular weight) from a solar composition (low mean molecular weight) atmosphere with uniform cloud cover.

\subsection{Application to Real Planets: HD $189733 b$ and HAT-P-11b}

We investigate the possibility of nonuniform cloud cover on two observed planets: the well-studied, canonical hot Jupiter HD 189733b, and the recently characterized warm Neptune HAT-P-11b.

We briefly summarize the previous conclusions inferred from the HST WFC3 transit observations of these planets. HD 189733b was observed with WFC3 in spatial scan mode by McCullough et al. (2014). Madhusudhan et al. (2014) presented the first extensive retrieval analysis of the HD 189733b HST WFC3 transmission spectra. They found, under the assumption of a cloud-free atmosphere, that a low water abundance was required to explain the spectra owing to the relatively muted features. They concluded that a high carbon-to-oxygen ratio was required to explain the apparent depletion of water.

A follow-up retrieval study of eight transmission spectra by Benneke (2015) also looked at HD 189733b. He compared the different abundance results (within a 1D self-consistent framework) that one obtains under the assumption of various degrees of cloud parameterizations, from the simple "Rayleigh Haze +opaque cloud deck" to parameterized profiles, assumed cloud compositions, and particle size distributions. He found, unsurprisingly, that with the addition of more free cloud parameters, the uncertainties in the abundances increased to a wider range of $\mathrm{C} / \mathrm{O}$ ratios, but still included $\mathrm{C} / \mathrm{O}>1$ within the $99.7 \%$ confidence interval.

HAT-P-11b (Bakos et al. 2010), a 900 K warm Neptune, was the first object in the warm-Neptune category to have claimed a water detection in transmission (Fraine et al. 2014). They concluded that a predominantly clear high-metallicity ( $\sim$ few tens $-300 \times$ solar at $1 \sigma$ confidence) atmosphere was most likely needed to explain the observed modulation.

We add a different interpretation to these two objects within the patchy cloud framework by performing identically the same analysis as was done on the synthetic data in Section 4.1. Figure 3 summarizes these results. As with the synthetic cases, 

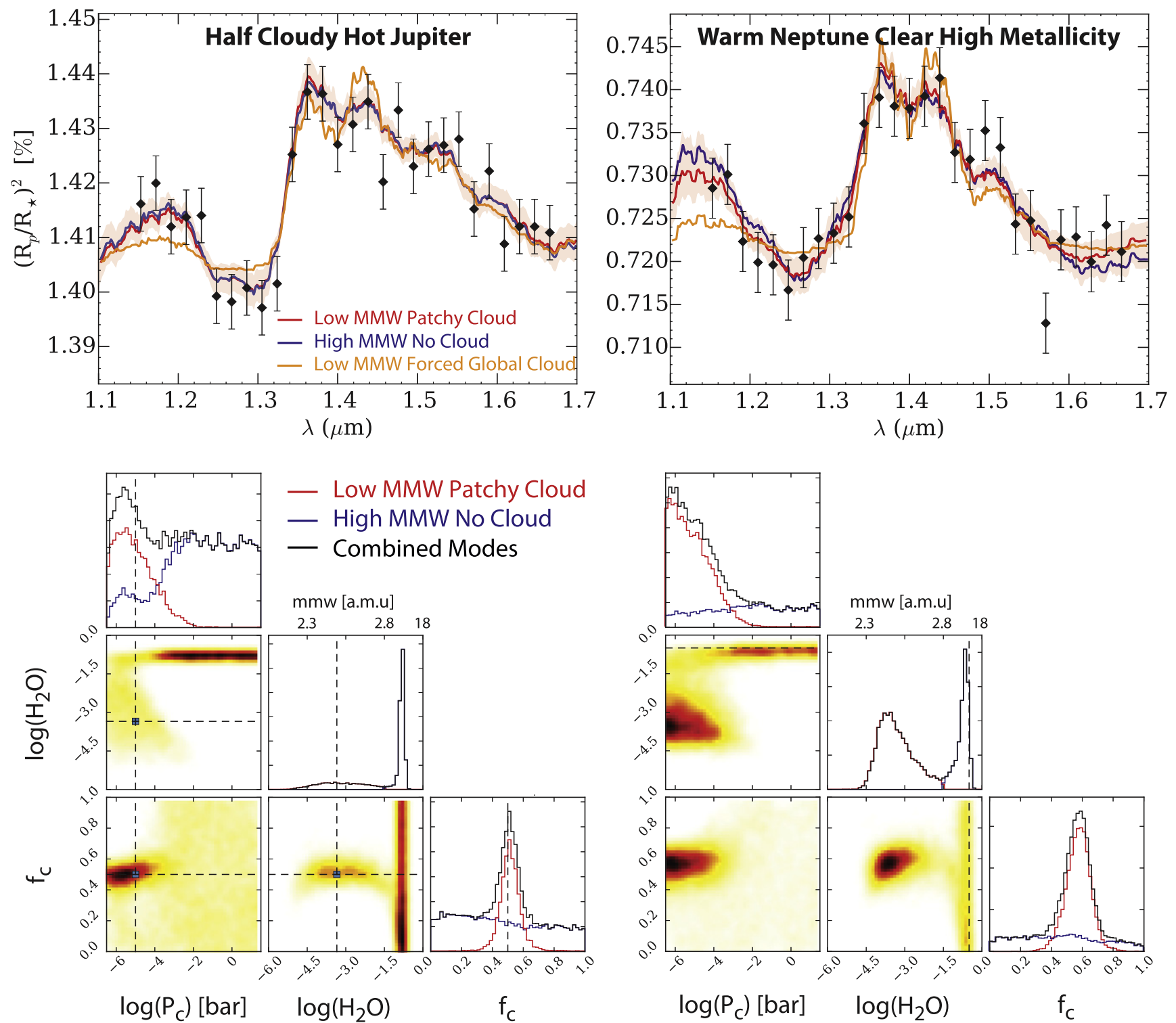

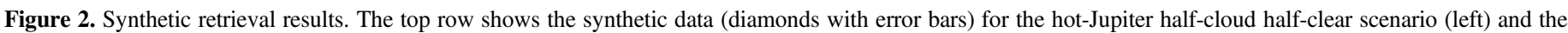
warm-Neptune high mean molecular weight scenario (right). For each the $2 \sigma$ spread in spectra derived from both modes is shown in light red. The median spectra drawn from the high and low water abundance modes only are shown in blue and red, respectively. The orange spectra are the median for a retrieval in which there is a globally uniform cloud and a prior upper limit restricting the high water abundance mode. The spectral modulation from one scale height is $150 \mathrm{ppm}$ in the solar composition hot Jupiter and $32 \mathrm{ppm}$ for the high molecular weight warm Neptune. The bottom row shows the posterior probability distributions for the interesting parameters (water abundance, cloud top pressure, and terminator cloud fraction). The black histograms are the resultant 1D marginalized distributions for each parameter. The red and blue curves result from drawing samples only from the low or high water (high molecular weight) modes, respectively (where the cut is at $\log \mathrm{H}_{2} \mathrm{O}=-1.5$, mean molecular weight of 2.8). The dashed lines show the true values for each scenario (the true cloud and cloud fraction for the warm-Neptune case are on the edges at 1.5 and 0 , respectively). Note that both scenarios can be fit well by either a high mean molecular weight or a partial cloud cover at approximately solar composition molecular weight. In the high mean molecular weight mode the cloud location and coverage are largely ill informed (blue histograms).

for both planets, we again find two possible solutions: the high mean molecular weight mode (resulting from the high water abundance) and the solar composition low mean molecular weight mode.

The correlations in Figure 3 are rich in information. For both planets, the red histograms, corresponding to the "patchy cloud" mode, always encompass a cloud fraction of unity (global cloud coverage), albeit at low probability, suggesting that some "clearness" is favored. Also in this patchy cloud mode, the cloud top pressures are required to be relatively low (high altitudes) for HD $189733 \mathrm{~b}\left(P_{\mathrm{c}}<\sim 1\right.$ mbar $)$ with a cloud fraction between 0.5 and 0.7 ( $1 \sigma$ width). The results are similar for HAT-P-11b, but less constrained owing to the lower feature signal-to-noise ratio. A more noticeable correlation between cloud top pressure and cloud fraction appears in HAT-P-11b. As the cloud top pressure decreases (higher altitudes), the cloud fraction must also decrease; otherwise, the water feature damps too much.

Looking at the $\log \left(\mathrm{H}_{2} \mathrm{O}\right)$ versus $\log \left(P_{c}\right)$ panels for both planets, we find that as the cloud moves to deeper pressures, the water abundance must decrease in order to preserve the lowamplitude features. These solutions correspond to the cloudfree "high C/O" solution found by Madhusudhan et al. (2014) for HD 189733b; less favorable fits in our models. The water features are damped when the water abundance is low because of the relative weighting of the water opacity to the hydrogen 

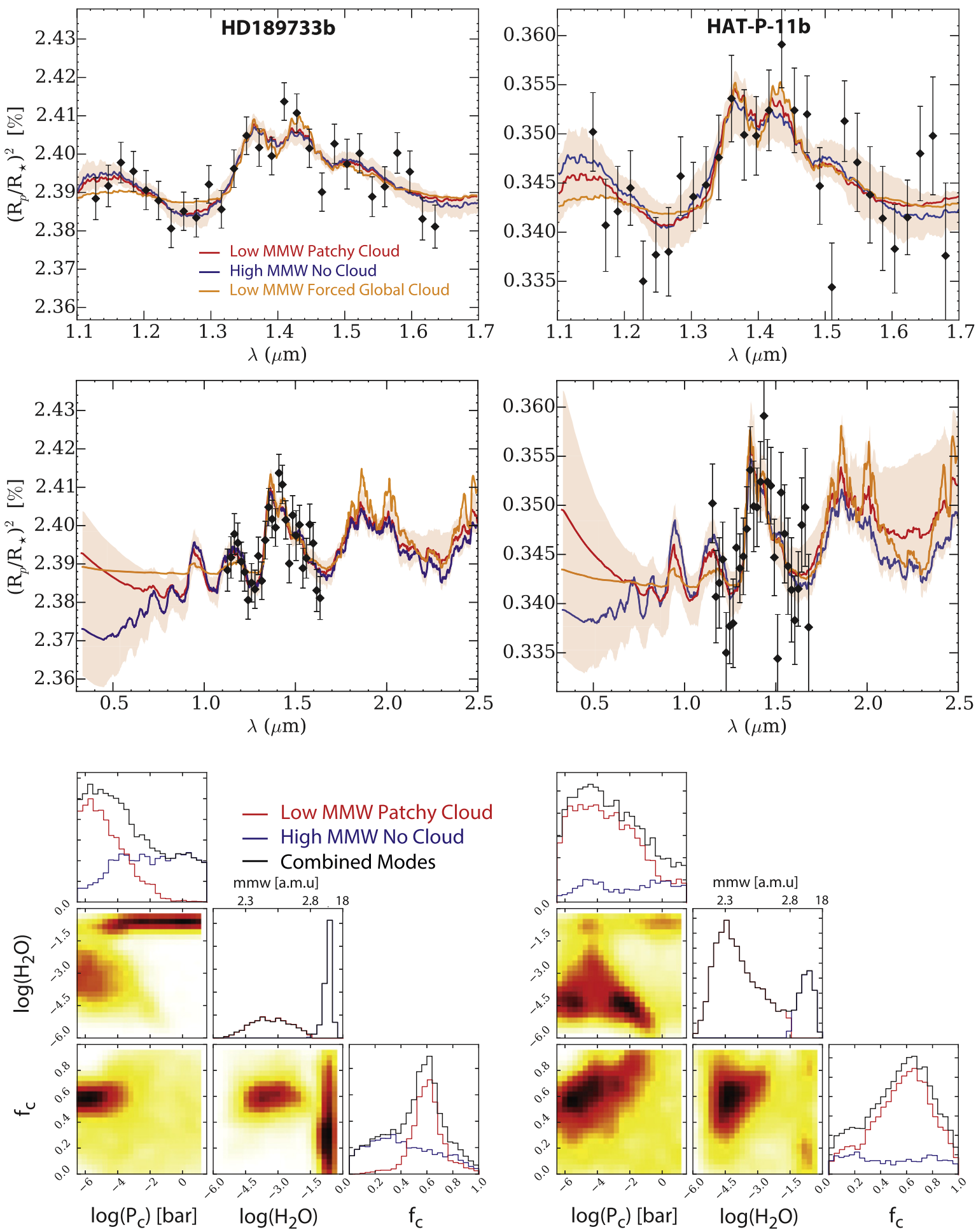

Figure 3. Retrieval results for HD $189733 \mathrm{~b}$ and HAT-P-11b. We have included only water, $\mathrm{H}_{2}$ Rayleigh scattering, $\mathrm{H}_{2}-\mathrm{H}_{2} / \mathrm{He}$ CIA, and gray clouds as absorbers. The top row shows the WFC3 data (diamonds with error bars) for HD $189733 \mathrm{~b}$ (left) and HAT-P-11b (right). For each, the $2 \sigma$ spread in spectra drawn from both modes is shown in light red. The median spectra drawn from the high and low water abundance modes are shown in blue and red, respectively. The orange curve in each is the median spectrum for a retrieval in which there is a globally uniform cloud and a prior upper limit restricting the high water abundance mode. The middle row shows a zoomed-out version from 0.3 to $2.5 \mu \mathrm{m}$. While all three scenarios are consistent with the HST WFC 3 data, they diverge significantly at shorter wavelengths. The bottom row shows the posterior probability distributions for the interesting parameters (water abundance, cloud top pressure, and terminator cloud fraction). The black histograms are the resultant 1D marginalized distributions for each parameter. The red and blue histograms result from drawing samples only from the low (mean molecular weights of 2.30-2.37 amu at 68\% confidence for HD 189733b and 2.30-2.30 amu for HAT-P-11b) or high (mean molecular weights of 4.52-6.92 amu at 68\% confidence for HD $189733 \mathrm{~b}$ and 3.38-7.94 amu for HAT-P-11b) water modes, respectively (where the cut is at $\log \mathrm{H}_{2} \mathrm{O}=-1.5$, mean molecular weight $=2.8 \mathrm{amu}$ ). The dashed lines show the true values for each scenario. Note that both scenarios can be fit well by either high mean molecular weight or fractional cloud cover at approximately solar abundances. In the high mean molecular weight mode the cloud location and coverage are largely ill informed (blue histograms) owing to the lack of sensitivity to those parameters at high water optical depths. 
CIA (Equation (8) with $\xi_{1} \sigma_{1}$ corresponding to water with $\xi_{2} \sigma_{2}$ corresponding to the CIA). This damping has a different behavior than a cloud as the CIA has a shape to it and is not spectrally flat. This increased CIA-to-water opacity due to the decreasing water abundance also explains why we can obtain a lower limit to the water mixing ratio constraint. A lower water abundance would result in pure CIA features.

For both objects we find that as the $\log \left(\mathrm{H}_{2} \mathrm{O}\right)$ increases to approximately $-1.5(3 \%)$, the noticeable change in the atmospheric mean molecular weight $(2.8 \mathrm{amu})$ begins to damp the spectral features (e.g., same as in Figure 6 of Benneke \& Seager 2013). Once the water abundance is high enough (blue histograms - high water abundance/mean molecular weight mode), the tangent optical depths reach unity at low enough pressures to be at altitudes above where the clouds appear to have an impact. This permits the cloud top pressure and hence cloud fraction to take on nearly any value (except for really low cloud top pressures).

Again, to be more quantitative, we undergo the same Bayes factor analysis as in Section 4.1. For HD 189733b, a weak to moderate favorability of the high-metallicity/mean molecular weight mode over the partly cloudy solar water mode $(\ln B=1.9)$ is found. Both the partly cloudly and highmetallicity modes are moderately favored over a solar composition with global cloud $\operatorname{cover}(\ln B=2.8)$. For HAT$\mathrm{P}-11 \mathrm{~b}$ all three scenarios are indistinguishable $(\ln B<\sim 1)$. This is an important point: The signal-to-noise ratio in the current WFC3 data for both HD 189733b and HAT-P-11b cannot definitively distinguish the difference between cloud-free highmetallicity atmospheres, solar composition patchy cloud atmospheres, and solar composition globally cloudy atmospheres. As shown with the numerical examples, and analytically, one cannot distinguish between high mean molecular weights and low mean molecular weight with fractional cloud cover over the WFC3 bandpass alone. However, high enough feature signal-to-noise ratios are enough to break the degeneracy between the low mean molecular weight partially cloudy or high mean molecular weight clear atmospheres and deeper global clouds.

If we look at predictions for other wavelengths (middle row, Figure 3) based on the fits to the WFC3 wavelengths, we can see some divergence in the different scenarios, especially at wavelengths $<1 \mu \mathrm{m}$. This is because of the relative weighting between the water opacity and the gaseous Rayleigh scattering. In the high mean molecular weight atmosphere the water absorption dominates over the molecular Rayleigh scattering, whereas in the patchy cloud solar abundance scenario, the Rayleigh scattering dominates over the water opacity (see the analytic discussion in Section 3). While shorter wavelengths can break the degeneracy between these three scenarios, the addition of a uniform high-altitude Rayleigh scattering haze layer, or additional opacity sources such as alkali metals, metal hydrides, and oxides, could potentially thwart our ability to do so. HD 189733 b has been observed from 0.3 to $1.0 \mu \mathrm{m}$ (Sing et al. 2009) and shows a strong slope with a large eclipse depth -much larger than the models presented here. The amplitude of the slope could be due either to scattering hazes (Sing et al. 2009) or to a lesser effect, starspots (McCullough et al. 2014). Strong scattering hazes in the optical could possibly make breaking the degeneracy between the three scenarios difficult. Despite these complications, however, it is apparent that the visible wavelengths offer the greatest chance of breaking this degeneracy.

HAT-P-11b was also observed in the Kepler bandpass. However, the broadband nature of those observations prevents a detailed characterization of the short-wavelength slopes, and uncertainties in the relative offsets between data sets observed at different epochs under different instrumental and astrophysical conditions may also muddle our ability to disentangle the three scenarios. Longer-wavelength spectra covering gases that present themselves more strongly at higher metallicities (e.g., $\mathrm{CO}, \mathrm{CO}_{2}$ ) should be able to break the high-metallicity versus patchy cloud scenario. In the next section we look to transit light curves as an observational signature for nonuniform cloud cover.

\section{IMPACT ON TRANSIT LIGHT CURVES}

During the eclipse phase of a transit, all the observed quantities are averaged over the planetary limb, leading to degeneracies in the transit spectrum. The shape of the ingress and the egress of the transit, however, is determined by the shape of the planetary limb and can potentially be used to constrain the cloud distribution over the planet limb and break the degeneracies between partial cloudiness and high mean molecular weight atmospheres.

We construct the transit light curve of a partially cloudy planet by combining the transit light curve of a planet with a clear atmosphere $\left(\psi_{\text {clear }}\right)$ with that of a cloudy atmosphere $\left(\psi_{\text {cloudy }}\right)$. The transit light curve of the annulus of a cloud can be constructed by subtracting the transit light curve of a clear planet from that of the cloudy planet: $\psi_{\text {ring }}=\psi_{\text {cloudy }}-\psi_{\text {clear }}$. We consider two extreme cases, corresponding to the two cloud-forming scenarios on tidally locked planets discussed in the introduction: the first is a planet with morning clouds only (i.e., no dependence on latitude but a dependence on longitude), and the second is a planet with polar clouds and a clear equator (i.e., no dependance on longitude but a dependence in latitude). In both cases we call $\alpha_{\mathrm{c}}$ the angle between the planet equator and the beginning of the cloudy evening terminator, counting from the morning side. The cloud coverage is therefore $f=1-\alpha_{c} / \pi$ for the morning cloud case and $f=1-2 \alpha_{c} / \pi$ for the polar cloud case. We call $\alpha$ the angle between the morning side of the planet equator and the intersection between the stellar and the planetary limb:

$$
\alpha=a \cos \left(\frac{p}{2 z}+\frac{z}{2 p}-\frac{1}{2 p z}\right)
$$

where $p$ is the ratio of the planetary radius to the stellar radius and $z$ is the apparent distance between the center of the star and the center of the planet divided by the stellar radius, as in Mandel \& Agol (2002). $\alpha$ is defined only during the ingress and the egress of the planet and goes from $\pi$ to 0 during both ingress and egress.

We now assume that the planet transits the stellar equator and that the planet is small compared to the star (e.g., the stellar luminosity is considered constant over the apparent size of the planet). Outside of transit, the transit light curve is $\psi=1$. During the full phase of the transit, the light curve is $\psi=\psi_{\text {clear }}+f \psi_{\text {ring. }}$. During the ingress, the phase curve can 


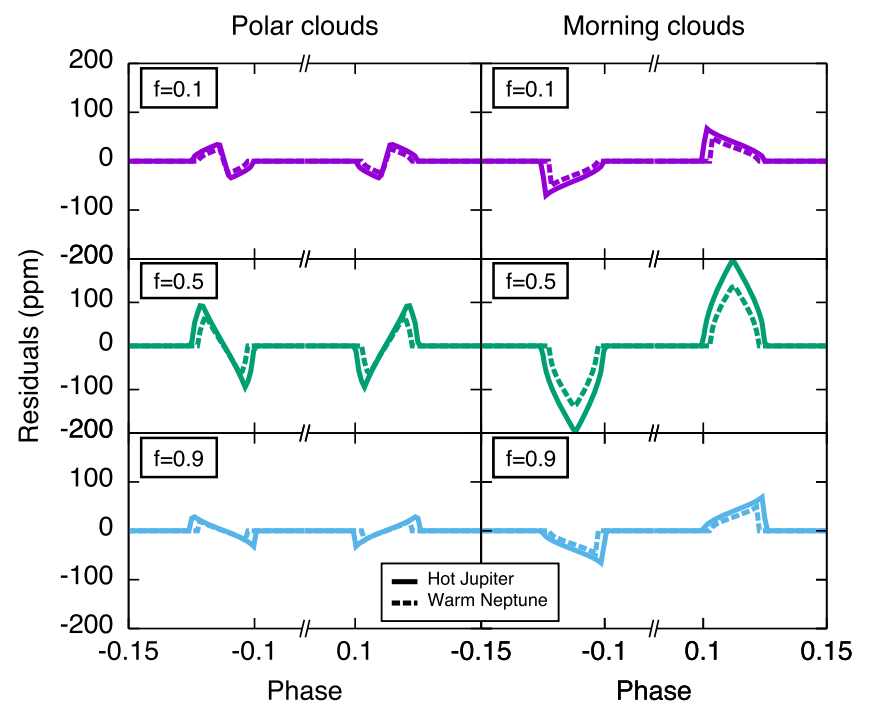

Figure 4. Relative difference between the transit light curve of a partially cloudy planet and the transit light curve of a spherical planet with the same apparent area. The left column is for a planet with banded clouds where the equator is cloudless and the poles are cloudy. The right column is for a planet with clouds on the morning terminator and clear on the evening terminator. The different rows are for different cloud fraction at the limb. Solid lines are for the synthetic hot-Jupiter scenario, and dotted lines for the synthetic warm-Neptune scenario. The difference in the effective opaque radius between the cloudy and clear terminators is of 5 scale heights. The light-curve residuals are directly proportional to this difference. We assumed no limb darkening and computed the cloudy and cloudless light curves with the occultsmall.f subroutine of Mandel \& Agol (2002).

be split into several parts. For morning clouds we have

$$
\psi= \begin{cases}\psi_{\text {clear }}+\psi_{\text {ring }}, & \text { for } \alpha>\alpha_{\mathrm{c}} \\ \psi_{\text {clear }}+\psi_{\text {ring }} \frac{\pi-\alpha_{\mathrm{c}}}{\pi-\alpha}, & \text { for } \alpha<\alpha_{\mathrm{c}}\end{cases}
$$

during the ingress and

$$
\psi= \begin{cases}\psi_{\text {clear }}+\psi_{\text {ring }} \frac{\alpha-\alpha_{\mathrm{c}}}{\alpha}, & \text { for } \alpha>\alpha_{\mathrm{c}} \\ \psi_{\text {clear }}, & \text { for } \alpha<\alpha_{\mathrm{c}},\end{cases}
$$

during the egress. For the polar cloud we have

$$
\psi= \begin{cases}\psi_{\text {clear }}, & \text { for } \alpha>\pi-\alpha_{\mathrm{c}} \\ \psi_{\text {clear }}+\psi_{\text {ring }} \frac{\pi-\alpha-\alpha_{\mathrm{c}}}{\pi-\alpha}, & \text { for } \alpha_{\mathrm{c}}<\alpha<\pi-\alpha_{\mathrm{c}} \\ \psi_{\text {clear }}+\psi_{\text {ring }} \frac{\pi-2 \alpha_{\mathrm{c}}}{\pi-\alpha}, & \text { for } \alpha<\alpha_{\mathrm{c}},\end{cases}
$$

during the ingress and

$$
\psi= \begin{cases}\psi_{\text {clear }}+\psi_{\text {ring }} \frac{\pi-2 \alpha_{\mathrm{c}}}{\alpha}, & \text { for } \alpha>\pi-\alpha_{\mathrm{c}} \\ \psi_{\text {clear }}+\psi_{\text {ring }} \frac{\alpha-\alpha_{\mathrm{c}}}{\alpha}, & \text { for } \alpha_{\mathrm{c}}<\alpha<\pi-\alpha_{\mathrm{c}} \\ \psi_{\text {clear }}, & \text { for } \alpha<\alpha_{\mathrm{c}},\end{cases}
$$

during the egress. The transit light curve of a partially cloudy planet can be computed by combining in the manner above the transit light curve of a cloudy and a cloudless planet, each computed separately via the IDL routines provided by Mandel \& Agol (2002).
Shown in Figure 4, the transit light curve of a partially cloudy planet will present residuals when modeled with a single uniform light curve. The amplitude of these residuals is $\sim 100 \mathrm{ppm}$. For these illustrative calculations, we assume that the effective radius of the cloudy planet is 5 scale heights larger than that of the clear, corresponding to clouds having an opacity $\approx 150$ times higher than the gas opacity in the bandpass of observation (Equation (1), Lecavelier Des Etangs et al. 2008). The residuals scale linearly with the difference in effective radius between the clear and cloudy atmospheres, thus with the difference between the natural $\log$ of the cloud and clear opacities. The signal should therefore be largest in bandpasses where the gaseous opacities are small.

As seen in Figure 4, the amplitude of the residuals is similar for the hot-Jupiter and warm-Neptune cases. This is because the residuals are proportional to the ratio of the areas of the cloudy annulus to the stellar disk. In our case the smaller scale height of our fiducial warm-Neptune model is compensated by the smaller radius of the star it transits (Table 1).

The shape of the residuals strongly depends on the distribution of clouds. Whereas the ingress and egress are symmetric in the case of polar clouds, they are antisymmetric in the case of morning clouds. A more complex picture is expected for more complex cloud distributions and could become degenerate with other effects such as planet oblateness (e.g., Carter \& Winn 2010).

Usually when interpreting transit light curves, both the planet and orbital parameters are fit simultaneously. This causes an additional degeneracy between the morning terminator cloud model and the center-of-transit time for a standard uniform limb model. We explored this degeneracy through some additional light-curve models. We found that the lightcurve residuals in Figure 4 are reduced by a factor of 10 given a shift in the transit timing of $\sim 10^{-5}$ and $\sim 10^{-4}$ days for a planet with a 1 and 10 scale height difference in radius between the clear and cloudy halves of the planet, respectively. We thus recommend high-precision transit timing to infer the presence of nonuniform cloud cover.

\section{DISCUSSION AND CONCLUSIONS}

We have demonstrated, both numerically and analytically, that nonuniform or "patchy" clouds" can impact the interpretation of transit transmission spectra. Albedo phase curve measurements (Demory et al. 2013; Esteves et al. 2015; Hu et al. 2015) and general circulation models with tracers (e.g., Parmentier et al. 2013; Charnay et al. 2015b) show strong inhomogeneities-both north-south and east-west-in the distribution of condensates in exoplanetary atmospheres. This suggests that the "patchy" cloud model is highly plausible.

We were able to show analytically why patchy terminator clouds, assuming that the cloudy terminator is dominated by a gray cloud, are able to exactly mimic small scale height atmospheres (typically due to high mean molecular weights) and why the spectral shape of patchy clouds is different from that of globally uniform clouds.

We find that for two well-observed planets, HD 189733b and HAT-P-11b, solar composition atmospheres with patchy terminator clouds can match the WFC3 transmission spectra just as well as other interpretations. Unfortunately, the signalto-noise ratio of the current WFC3 data for these two objects is not high enough to permit a definitive differentiation among cloud-free high mean molecular weight atmospheres, 
solar composition patchy cloud atmospheres with a highaltitude $\left(P_{c}<1\right.$ mbar) cloud, and solar composition mid-level $\left(0.1 \mathrm{mbar}<P_{c}<100\right.$ mbar) globally cloudy atmospheres. This is in contrast to our synthetic example, where we could differentiate the nonuniform cloud cover and global cloud coverage (but not high metallicity), unsurprising given that the synthetic feature-to-noise ratios are nearly twice as large. Obtaining a similar feature signal-to-noise ratio for HD $189733 \mathrm{~b}$ and HAT-P-11b should allow us to rule out or confirm the global cloud scenario as parameterized here. To distinguish the high-metallicity/mean molecular weight case from the solar patchy cloud case, one would have to identify high-metallicity features at longer wavelengths due to $\mathrm{CO}$ and $\mathrm{CO}_{2}$ or look at shorter wavelengths as shown in Figure 3. We also found that patchy terminator clouds can result in lightcurve residuals of up to a couple of hundred ppm, if the transit timing is known to seconds.

In addition to these two differentiating observations, one may also use prior information such as interior structure model estimates of the bulk metallicity given the measured mass and radius. However, for highly irradiated hot Jupiters, such as HD 189733b, this task is difficult because there exists a degeneracy between the bulk metal content and the yet to be definitively determined "inflation" mechanisms (e.g., Batygin et al. 2011; Miller \& Fortney 2011; Thorngren et al. 2015), and it is not yet clear how the bulk metal content partitions itself between the core and envelope.

One may question the plausibility of nonuniform cloud cover on cooler planets. Cooler planets, in general, are expected to have more homogenous day-night temperature contrasts, effectively removing nightside cold traps in which classical equilibrium condensates can form. However, we can envision a variety of scenarios in which this may not necessarily matter. For instance, photochemical precursers could be produced on the dayside of the planet, but could continue to polymerize through radical transport on the nightside without being further destroyed by the UV flux. Furthermore, Kataria et al. (2014) showed that day-night contrasts can reach up to a few hundred kelvin at low pressures on warm Neptune-like planets (e.g., GJ 1214b), typically where photochemical hazes are formed (e.g., Morley et al. 2013; 2015). Additionally, most inhomogeneous cloud cover in the atmospheres of solar system bodies is driven by meridional temperature gradients owing to the north-south insolation gradient rather than day-to-night temperature gradients. In essence, theoretically, patchy cloud terminators in transiting planets can be common.

Another interesting aspect of the 3D problem is that of scale height variations from one terminator to the next in highly irradiated planets, as explored in Fortney et al. (2010) and Burrows et al. (2010). Could variations in scale height be degenerate with nonuniform cloud cover? If we assume a morning-evening temperature difference of $500 \mathrm{~K}$, a reasonable temperature gradient, we find only 3\%-5\% fractional annulus area change compared to a half-cloudy half-clear model that gives a 5\%-11\% fractional area difference. We should be mindful of this possible additional degeneracy as the larger scale height will occur on the evening terminator of the planet, whereas cloud formation will likely occur on the morning. The scale height variation may potentially damp the amplitude of variation due to the patchy clouds by up to half.

Finally, in order to fully address the complicated nature of clouds, future investigations should consider the full three- dimensional aspect of the problem and a broader range of cloud prescriptions (as opposed to the simple cloud top pressure or uniform gray absorber parameterizations). Computing 3D transmission spectra from GCMs with clouds of varying particle sizes/compositions will offer more insight into the possible complications when interpreting transit transmission spectra. Future higher signal-to-noise ratio observations covering a broader range of wavelengths will likely be able to spectroscopically decipher the differences between high metallicity, uniform cloud cover, and patchy clouds. Finally, looking at an ensemble of planets over a wide range of temperatures will also aid in deciphering the role of nonuniform cloud cover, similar to what has been done with brown dwarfs.

We thank Jonathan Fortney and Mark Swain for reading the manuscript and providing useful comments, and Daniel Thorngren, Leslie Rogers, and Caroline Morley for useful discussions. M.R.L. acknowledges support provided by NASA through Hubble Fellowship grant 51362 awarded by the Space Telescope Science Institute, which is operated by the Association of Universities for Research in Astronomy, Inc., for NASA, under the contract NAS 5-26555. V.P. acknowledges support from the Sagan Postdoctoral Fellowship through the NASA Exoplanet Science Institute. The simulations for this research were carried out on the UCSC Hyades computing cluster, which is supported by National Science Foundation (award number AST-1229745) and University of California, Santa Cruz.

\section{REFERENCES}

Ackerman, A. S., \& Marley, M. S. 2001, ApJ, 556, 872

Apai, D., Radigan, J., Buenzli, E., et al. 2013, ApJ, 768, 121

Bakos, G. Á., Torres, G., Pál, A., et al. 2010, ApJ, 710, 1724

Batygin, K., Stevenson, D. J., \& Bodenheimer, P. H. 2011, ApJ, 738, 1

Benneke, B. 2015, arXiv:1504.07655

Benneke, B., \& Seager, S. 2012, ApJ, 753, 100

Benneke, B., \& Seager, S. 2013, ApJ, 778, 153

Brown, T. M. 2001, ApJ, 553, 1006

Buchner, J., Georgakakis, A., Nandra, K., et al. 2014, A\&A, 564, A125

Buenzli, E., Apai, D., Morley, C. V., et al. 2012, ApJL, 760, L31

Buenzli, E., Apai, D., Radigan, J., Reid, I. N., \& Flateau, D. 2014, ApJ, 782, 77

Burgasser, A. J., Marley, M. S., Ackerman, A. S., et al. 2002, ApJL, 571, L151

Burrows, A., Rauscher, E., Spiegel, D. S., \& Menou, K. 2010, ApJ, 719, 341

Burrows, A., Sudarsky, D., \& Hubeny, I. 2006, ApJ, 640, 1063

Carter, J. A., \& Winn, J. N. 2010, ApJ, 716, 850

Charnay, B., Meadows, V., \& Leconte, J. 2015a, ApJ, 813, 15

Charnay, B., Meadows, V., Misra, A., Leconte, J., \& Arney, G. 2015b, ApJL, 813, L1

Cornish, N. J., \& Littenberg, T. B. 2007, PhRvD, 76, 083006

Cowan, N. B., Machalek, P., Croll, B., et al. 2012, ApJ, 747, 82

Crossfield, I. J. M., Biller, B., Schlieder, J. E., et al. 2014, Natur, 505, 654

Crossfield, I. J. M., Hansen, B. M. S., Harrington, J., et al. 2010, ApJ, 723, 1436

Cushing, M. C., Marley, M. S., Saumon, D., et al. 2008, ApJ, 678, 1372

Deming, D., Wilkins, A., McCullough, P., et al. 2013, ApJ, 774, 95

Demory, B.-O., de Wit, J., Lewis, N., et al. 2013, ApJL, 776, L25

de Wit, J., \& Seager, S. 2013, Sci, 342, 1473

Esteves, L. J., De Mooij, E. J. W., \& Jayawardhana, R. 2015, ApJ, 804, 150

Feroz, F., Hobson, M. P., \& Bridges, M. 2009, MNRAS, 398, 1601

Fortney, J. J. 2005, MNRAS, 364, 649

Fortney, J. J., Shabram, M., Showman, P., et al. 2010, ApJ, 709, 1396

Fraine, J., Deming, D., Benneke, B., et al. 2014, Natur, 513, 526

Griffith, C. A. 2014, RSPTA, 372, 20130086

Howe, A. R., \& Burrows, A. S. 2012, ApJ, 756, 176

Hu, R., Demory, B.-O., Seager, S., Lewis, N., \& Showman, A. P. 2015, ApJ, 802,51

Kataria, T., Showman, A. P., Forthey, J. J., et al. 2015, ApJ, 801, 86

Kirkpatrick, J. D. 2005, ARA\&A, 43, 195 
Knutson, H. A., Benneke, B., Deming, D., \& Homeier, D. 2014, Natur, 505, 66 Knutson, H. A., Charbonneau, D., Allen, L. E., et al. 2007, Natur, 447, 183

Knutson, H. A., Charbonneau, D., Cowan, N. B., et al. 2009, ApJ, 703, 769

Knutson, H. A., Lewis, N., Fortney, J. J., et al. 2012, ApJ, 754, 22

Kreidberg, L., Bean, J. L., Désert, J.-M., et al. 2014a, ApJL, 793, L27

Kreidberg, L., Bean, J. L., Désert, J.-M., et al. 2014b, Natur, 505, 69

Kreidberg, L., Line, M. R., Bean, J. L., et al. 2015, ApJ, in press (arXiv:1504.05586)

Lecavelier Des Etangs, A., Pont, F., Vidal-Madjar, A., \& Sing, D. 2008, A\&A, 481, L83

Lewis, N. K., Knutson, H. A., Showman, A. P., et al. 2013, ApJ, 766, 95

Line, M. R., Knutson, H., Deming, D., Wilkins, A., \& Desert, J.-M. 2013, ApJ, 778,183

Madhusudhan, N., Crouzet, N., McCullough, P. R., Deming, D., \& Hedges, C. 2014, ApJL, 791, L9

Mandel, K., \& Agol, E. 2002, ApJL, 580, L171

Marley, M. S., \& Robinson, T. D. 2015, ARA\&A, 53, 279

Marley, M. S., Saumon, D., \& Goldblatt, C. 2010, ApJL, 723, L117

Morley, C. V., Fortney, J. J., Kempton, E. M.-R., et al. 2013, ApJ, 775, 33

Morley, C. V., Fortney, J. J., Marley, M. S., et al. 2015, ApJ, 815, 110

McCullough, P. R., Crouzet, N., Deming, D., \& Madhusudhan, N. 2014, ApJ, 791,55
Metchev, S. A., Heinze, A., Apai, D., et al. 2015, ApJ, 799, 154

Miller, N., \& Fortney, J. J. 2011, ApJL, 736, L29

Morley, C. V., Fortney, J. J., Kempton, E. M.-R., et al. 2013, ApJ, 775, 33

Morley, C. V., Marley, M. S., Fortney, J. J., et al. 2014, ApJ, 787, 78

Parmentier, V., Fortney, J. J., Showman, A. P., Morley, C. V., \& Marley, M. S. 2016, arXiv: 1602.03088

Parmentier, V., Showman, A. P., \& Lian, Y. 2013, A\&A, 558, A91

Radigan, J., Jayawardhana, R., Lafrenière, D., et al. 2012, ApJ, 750, 105

Radigan, J., Lafrenière, D., Jayawardhana, R., \& Artigau, E. 2014, ApJ, 793, 75

Showman, A. P., \& Guillot, T. 2002, A\&A, 385, 166

Shporer, A., \& Hu, R. 2015, AJ, 150, 112

Sing, D. K., Désert, J.-M., Lecavelier Des Etangs, A., et al. 2009, A\&A, 505,891

Stevenson, K. B., Désert, J.-M., Line, M. R., et al. 2014, Sci, 346, 838

Swain, M. R., Line, M. R., \& Deroo, P. 2014, ApJ, 784, 133

Thorngren, D. P., Fortney, J. J., \& Lopez, E. D. 2015, ApJ, submitted (arXiv:1511.07854)

Tinetti, G., Tennyson, J., Griffith, C. A., \& Waldmann, I. 2012, RSPTA, 370, 2749

Trotta, R. 2008, ConPh, 49, 71

Vahidinia, S., Cuzzi, J. N., Marley, M., \& Fortney, J. 2014, ApJL, 789, L11

Zhang, X., \& Showman, A. P. 2014, ApJL, 788, L6 